\title{
Fístulas del seno piriforme congénitas. Reporte de 4 casos y revisión de la literatura
}

\author{
Daniel Rappoport W. ${ }^{1}$, Nicolas von Jentschyk R. ${ }^{1}$, Patricio Gac E. ${ }^{1}$, \\ Francisco Rodríguez M. ${ }^{1}$ y Patricio Cabane T. ${ }^{1}$
}

'Departamento de Cirugía Hospital Clínico Universidad de Chile. Santiago, Chile.

Recibido el 25 de noviembre de 2016 y aceptado el 26 de enero de 2017

Correspondencia a: Dr. Daniel Rappoport W. dr.rappoport@gmail.com rappoport@u.uchile.cl

\section{Congenital pyriform sinus fistula. Report of 4 cases and review of the literature}

Introduction: Pyriform sinus fistula is a rare cervical branchial anomaly derived from the third and fourth branchial arch. Pyriform sinus fistula should be considered in children presenting enlarged neck mass, fistula, abscess, thyroid infection and/or dysphonia. Objective: We here report 4 cases treated in Hospital Clínico Universidad de Chile and review of the current literature on pyriform sinus fistula. Discussion: When clinical presentation is compatible with pyriform sinus fistula, endoscopic and imaging techniques are required. Surgical outcomes are better, although endoscopic therapies and combination of both surgery and endoscopy have also reported good outcomes. Conclusion: Diagnosis is made based on signs and symptoms and a high index of clinical suspicion. Open surgery is gold standard, however in pediatric population endoscopic resolution should be considered to reduce the high morbidity of traditional surgery. Key words: pyriform sinus fistula; branchial arch anomalies.

\section{Resumen}

Introducción: Las fístulas del seno piriforme, originadas desde el tercer o cuarto arco branquial, son entidades poco frecuentes. Se pueden presentar como aumento de volumen cervical, fístula cervical, absceso cervical, tiroiditis abscedada y/o disfonía. Objetivo: Reportar 4 casos tratados en nuestro hospital y revisión de la literatura. Discusión: Frente a un cuadro clínico compatible, se debe realizar estudio endoscópico y de imágenes. El manejo quirúrgico sigue siendo el de mejores resultados, pero se han desarrollado terapias endoscópicas y combinaciones de estas con buenos resultados. Conclusión: El diagnóstico requiere un alto índice de sospecha clínica. La cirugía abierta se mantiene como el gold standard, sin embargo, en población pediátrica estaría indicada la resolución endoscópica debido a la alta morbilidad de la cirugía tradicional.

Palabras clave: fístulas del seno piriforme; anomalías de los arcos branquiales.

\section{Introducción}

Las anomalías del desarrollo branquial representan alrededor del 17\% de las masas cervicales en la población pediátrica. De estas, las más frecuentes se originan en el segundo arco branquial, seguidas en segundo lugar, por las del primer arco y solo el $3-10 \%$ corresponderían a anomalías del tercer y cuarto arcos branquiales ${ }^{1}$. La alteración en el desarrollo embrionario se puede traducir en un quiste, seno o fístula branquial, dependiendo del tipo de falla en la obliteración de este. Al ser una malformación congénita, su diagnóstico generalmente es en niños, pero al tratarse de una entidad de baja fre- cuencia, y que además puede mantenerse asintomática por largo tiempo, es por lo que su diagnóstico se realiza en adultos hasta en la mitad de los casos ${ }^{2}$. Para esto se requiere de una alta sospecha clínica. El propósito de este trabajo es el reporte de 4 casos tratados en el Hospital Clínico de la Universidad de Chile y una revisión de la literatura publicada sobre esta entidad.

\section{Pacientes y Método}

Trabajo descriptivo retrospectivo, incluyendo todos los pacientes con fístulas del seno piriforme 
tratados en el Hospital Clínico de la Universidad de Chile durante un período de 10 años (2006-2016). Para esto se revisó el registro institucional por diagnóstico de egreso hospitalario, y de estos, se hizo una revisión de la ficha clínica. Además se realizó una revisión de la literatura tanto en español como en inglés sobre el tema.

\section{Casos}

\section{Caso $n^{o} 1$}

Paciente de 15 años, de sexo femenino, quien consulta por cuadro de 10 días de evolución caracterizado por aumento de volumen cervical izquierdo, sensible, asociado a fiebre de $38^{\circ} \mathrm{C}$, cefalea y rigidez cervical. Se indica tratamiento antibiótico con amoxicilina y ácido clavulánico por 10 días, con mala respuesta, por lo que consulta al servicio de urgencias. Se solicita tomografía computarizada cervical en la que destaca absceso de $3,2^{\prime} 3 \mathrm{~cm}$, entre el seno piriforme izquierdo y el polo superior del lóbulo tiroideo ipsilateral (Figura 1). Se realiza drenaje quirúrgico asociado a tratamiento antibiótico intravenoso; 48 h más tarde se realiza exploración cervical, resección del quiste y cierre de la fístula. Evoluciona favorablemente por lo que se indica alta al segundo día posoperatorio. Biopsia compatible con lesión quística abscedada, sin malignidad en lo examinado. La paciente se encuentra asintomática, sin evidencias de recidiva en 63 meses de seguimiento.

\section{Caso $n^{\circ} 2$}

Paciente de 20 años, de sexo masculino, presenta absceso cervical izquierdo con ecografía cervical y tomografía computarizada de cuello compatibles con absceso peritiroideo izquierdo con prolongación a seno piriforme ipsilateral. Se indica tratamiento antibiótico con amoxicilina y ácido clavulánico por 14 días, con buena respuesta. Se realiza tomografía computarizada de control 2 años y medio después, que resulta sin imágenes sugerentes de fístula residual u otra lesión; el esofagograma no muestra extravasación de medio de contraste y en el último control, 72 meses después, el paciente se encuentra asintomático y sin aumento de volumen al examen clínico.

\section{Caso $n^{\circ} 3$}

Paciente de 23 años, de sexo femenino, con antecedentes de shock séptico secundario a absceso cérvico-mediastínico drenado en otro centro un año previo a la consulta. Además, presenta antecedente

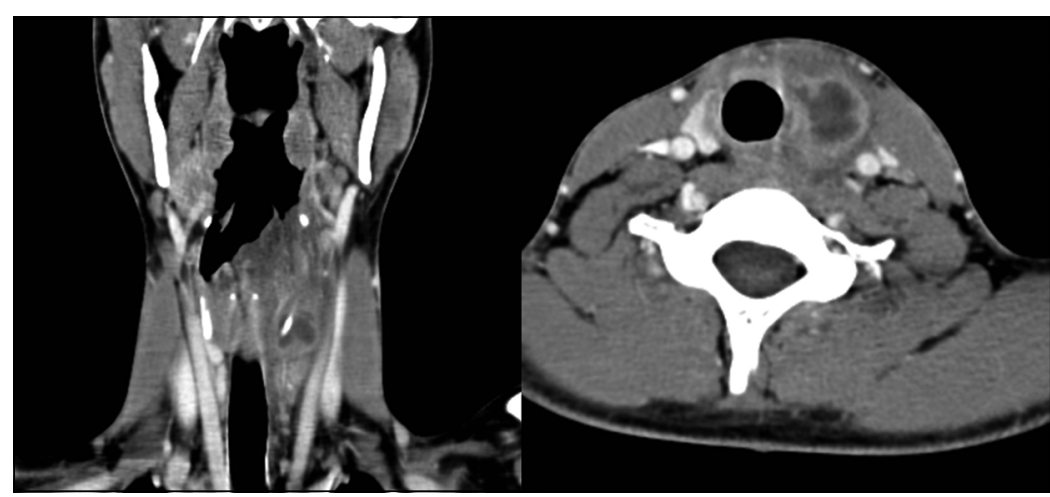

Figura 1. Tomografía computarizada del cuello con contraste intravenoso del caso $n^{\circ} 1$. Cortes coronal y axial en los que se evidencia absceso en relación con seno piriforme izquierdo y polo superior de lóbulo tiroideo izquierdo.

de trombofilia (déficit de proteínas $\mathrm{C}$ y S) y embarazo de 23 semanas al momento en que consulta por aumento de volumen cervical izquierdo doloroso. Es evaluada en el servicio de urgencias, donde se solicita eco cervical que muestra absceso peritiroideo izquierdo de $2 \mathrm{~cm}$ de diámetro. Se indica tratamiento antibiótico; consulta a las $48 \mathrm{~h}$ por mala respuesta; se objetiva crecimiento de la lesión a $3 \mathrm{~cm}$ que impresiona, en esta oportunidad, de localización intratiroidea izquierda; además presenta alza de parámetros inflamatorios, por lo que se decide la hospitalización y el manejo con tratamiento antibiótico intravenoso con ceftriaxona asociada a clindamicina. La paciente evoluciona con buena respuesta clínica, la cual se objetiva además con una nueva ecografía cervical que muestra la reducción de la lesión a $1 \mathrm{~cm}$. Es evaluada por infectología, quienes deciden mantener profilaxis antibiótica secundaria con amoxicilina hasta la resolución. Seis meses después -3 meses posparto- acude a control, refiere disfagia lateral derecha, sin molestias a izquierda. Se solicita resonancia nuclear magnética, que no muestra lesión residual ni imágenes sugerentes de fístula. Desde el punto de vista clínico, actualmente se encuentra asintomática y permanece con profilaxis secundaria hasta el último control a los 9 meses.

\section{Caso $n^{\circ} 4$}

Paciente de 18 años, de sexo femenino, con antecedente de absceso cervical profundo 2 años previos a la consulta y diagnóstico de adenitis de probable foco odontogénico, que requirió drenaje quirúrgico. A los 5 meses previos al ingreso presenta pequeño aumento de volumen en la base de cuello izquierdo, que evoluciona con gran compromiso del estado general, fiebre, calofríos, contractura cervical y cervicalgia. Se hospitaliza en esa oportunidad por sepsis 
de foco cervical secundario a absceso profundo del cuello, requiriendo drenaje percutáneo por radiología intervencional asociado a tratamiento antibiótico con amoxicilina, el que se mantiene como profilaxis secundaria hasta la resolución.

Se realiza estudio de manera ambulatoria con tomografía computarizada del cuello, que evidencia imagen compatible con fístula del seno piriforme izquierdo y esofagograma que confirma el diagnóstico (Figura 2). Se realiza la primera intervención en nuestro centro con resección de la fístula del seno piriforme, que se envía a biopsia rápida compatible con trayecto fistuloso e inflamación crónica perilesional; evoluciona favorablemente por lo que se indica el alta al segundo día. La biopsia definitiva confirma el diagnóstico. Clínicamente asintomática, el control con esofagograma 5 meses después informa de hallazgos compatibles con fístula de escaso débito del seno piriforme izquierdo. Se decide nueva intervención, con doble abordaje: abierto y endoscópico. En esta ocasión, a través de laringoscopia directa, en la base del seno piriforme izquierdo se logra evidenciar una apertura de fístula de $1 \mathrm{~mm}$, la cual se cánula. Por cervicotomía anterior se identifican estructuras y cánulas, se

Figura 2. Esofagograma preoperatorio del caso $n^{\circ} 4$, en el que se evidencia extravasación de medio de contraste desde el seno piriforme izquierdo y trayecto fistuloso. La flecha amarilla indica el final de trayecto fistuloso.

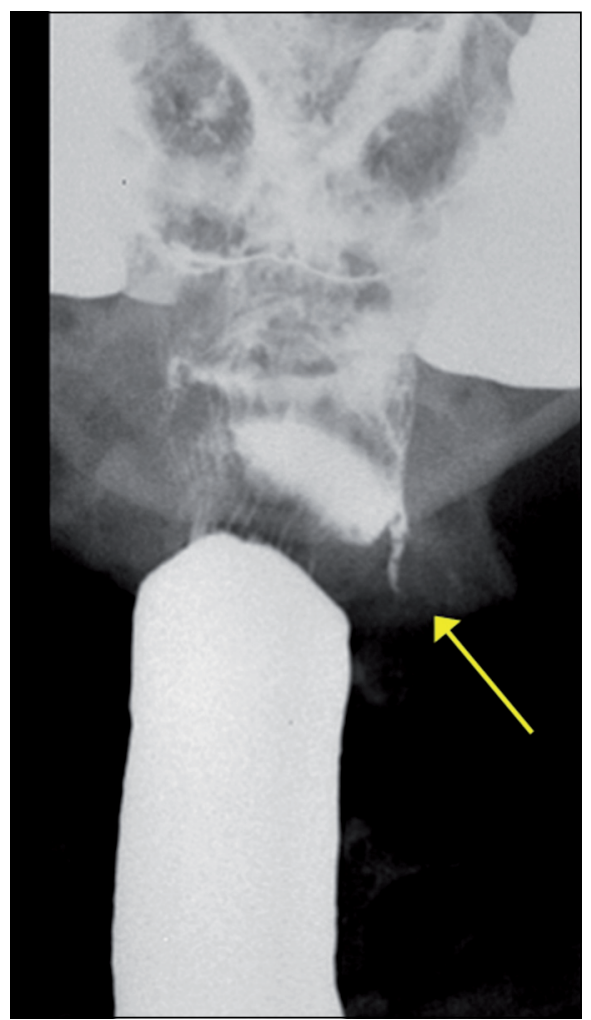

reseca el remanente asociado a lobectomía tiroidea izquierda y se practica refuerzo del cierre de fístula con colgajo de esternocleidomastoideo. La paciente evoluciona favorablemente y se da de alta al segundo día posoperatorio. El control con esofagograma un mes después se muestra sin evidencia de fístula residual, y a los 10 meses de seguimiento la paciente se encuentra asintomática.

\section{Discusión}

Las fístulas del seno piriforme son una entidad poco frecuente, por lo que requieren una alta sospecha clínica, para poder llegar a su diagnóstico ${ }^{1,3,4}$. Estas se presentan en un $95 \%$ de los casos a la izquierda y clínicamente se pueden presentar como aumento de volumen cervical, fístula cervical, absceso cervical, tiroiditis abscedada y/o disfonía ${ }^{1,4}$. Por lo que debemos tener como diagnóstico diferencial, ante estos cuadros clínicos, la posibilidad de una fístula de tercer o cuarto arco.

Estas fístulas se originan en el seno piriforme. Las del tercer arco lo hacen en la base del seno, atraviesan la membrana tirohioidea, a través o adosada al lóbulo tiroideo, profundo al nervio glosofaríngeo, pero superficial al laríngeo superior ${ }^{4}$. En cambio, las que se originan en el cuarto arco parten del ápex del seno, con un trayecto similar pero profundo al nervio laríngeo superior y superficial al nervio laríngeo recurrente, pudiendo completar el trayecto de este nervio ${ }^{3}$.

Una vez sospechada, se debe iniciar el estudio, el cual se basa en los estudios endoscópicos e imágenes. En el estudio endoscópico, el fin es certificar la apertura en el seno piriforme y objetivar si esta se localiza en la base o en el ápex de este, con un VPP del $90 \%{ }^{4}$. Esto permite diferenciar las fístulas del tercer arco de las del cuarto arco.

Con respecto a las imágenes, habitualmente se inicia el estudio con una ecografía, la que va a mostrar hallazgos inespecíficos con un VPP del 33\%. En un esofagograma, se puede apreciar la extravasación del medio de contraste, contrastando a veces el trayecto completo del seno o fístula, con un VPP del $88-100 \%{ }^{4}$. Esto nos permite no solo realizar el diagnóstico, sino también orientar el trayecto y la magnitud de la resección necesaria para resolver de manera correcta el cuadro. Es muy importante diferir este estudio al menos de 6 a 8 semanas luego de una sobreinfección, ya que la inflamación podría obstruir la apertura en el seno, dando un falso negativo al examen ${ }^{5}$. La tomografía computarizada también tiene un rol importante ya que nos puede 
mostrar en forma tridimensional aire en el posible trayecto fistuloso, extensión del absceso, pérdida de la rápida atenuación del contraste del tiroides, obliteración del seno piriforme, compromiso mediastínico, etc. ${ }^{6}$. Es importante resaltar que esta técnica se enriquece del uso de medio de contraste intravenoso y oral, logrando con este último una fistulografía tridimensional cuando se contrasta la fístula, alcanzado un VPP de hasta un $88 \%{ }^{5}$. Hay otros que prefieren el uso de resonancia magnética, la que podría discriminar mejor en partes blandas la zona de la fístula, absceso, etc., pero con un VPP inferior del orden del $63 \%{ }^{6}$.

Una vez realizado el diagnóstico, se debe definir el tratamiento, que puede ser: conservador, endoscópico, quirúrgico o combinaciones de estos. El manejo conservador se basa en el uso de antiinflamatorios asociados a antibióticos. Estos pueden ser de amplio espectro, o enfocados a los agentes más frecuentes, que pueden variar según la población. Ahn et al. ${ }^{2}$ demostraron, en una población coreana, que las bacterias más frecuentes en los cultivos de niños eran estreptococos grampositivos, en cambio, en los adultos era una bacteria gramnegativa, Klebsiella pneumoniae.

El tratamiento endoscópico se basa en el cierre de la apertura de esta. Eso habitualmente se logra con la cauterización de la apertura en el seno, ya sea con electrocauterio, ácido tricloroacético, nitrato de plata o Fibrin glue ${ }^{\circledR}$, con tasas de éxito del $75 \%{ }^{7,8}$. Este tipo de tratamiento ha sido muy popular en niños, especialmente en menores de 8 años. Esto porque tiene cifras aceptables de éxito, con una baja morbilidad. En este subgrupo, la morbilidad de la cirugía abierta puede llegar hasta el $10 \%$, la cual baja considerablemente en pacientes mayores de 8 años, en los que llega a menos del $2 \%{ }^{4}$. Una modificación de esta técnica la hicieron Josephson y Black ${ }^{1}$, quienes describieron la cauterización asociada a la sutura endoscópica de esta en un caso.

El tratamiento quirúrgico abarca desde el drenaje solamente, con tasas de falla cercanas al 100\%, a la fistulectomía abierta, con tasas de éxito del $85 \%$ que suben al 92\% al asociar la hemitiroidectomía ipsilateral ${ }^{4}$. El grupo de Givens et al. ${ }^{9}$ asocian a esta técnica un colgajo de esternotiroideo de base superior, que lo rotan para reforzar el cierre de la apertura en el seno, sin recidivas en este grupo de pacientes.

Se ha descrito la combinación de técnicas endoscópicas asociadas a la cirugía en diferentes modalidades. Arunachan et al. describen la cauterización endoscópica asociada a la fistulectomía y hemitiroidectomía abiertas en solo 5 casos, pero sin recidivas al cabo de 3 años ${ }^{10}$.
En nuestra serie, hay 2 pacientes que se resolvieron quirúrgicamente. En uno de ellos hubo que reintervenir, en esa oportunidad, el aporte del apoyo endoscópico para objetivar la apertura, y el cierre de esta misma durante la cirugía abierta fue fundamental para el éxito. En los 2 casos que se resolvieron con manejo conservador, llama la atención que en los controles posteriores, no se ha podido demostrar la fístula, lo que plantea la duda de si, secundaria a la infección, la inflamación habría sido capaz de obliterar la fístula.

\section{Conclusión}

Las fístulas del seno piriforme son una entidad poco frecuente, formando parte del espectro de las anomalías del desarrollo de los arcos branquiales. Su diagnóstico requiere una alta sospecha clínica.

La cirugía abierta sigue siendo el gold standard del tratamiento, sin embargo, en la población pediátrica, en especial en los menores de 8 años, se prefieren los tratamientos endoscópicos, ya que a pesar de tener tasas de éxito menores, la cirugía abierta tiene una elevada morbilidad en este subgrupo de pacientes.

\section{Responsabilidades éticas}

Protección de personas y animales. Los autores declaran que para esta investigación no se han realizado experimentos en seres humanos ni en animales.

Confidencialidad de los datos. Los autores declaran que han seguido los protocolos de su centro de trabajo sobre la publicación de datos de pacientes.

Derecho a la privacidad y consentimiento informado. Los autores han obtenido el consentimiento informado de los pacientes y/o sujetos referidos en el artículo. Este documento obra en poder del autor de correspondencia.

\section{Financiación}

Los autores declaran no haber recibido ninguna financiación para la realización de este trabajo.

\section{Conflicto de intereses}

Los autores declaran no tener ningún conflicto de intereses. 


\section{CASO CLÍNICO}

\section{Bibliografía}

1. Josephson G, Black K. A review over the past 15 years of the management of the internal piriform apex sinus tract of a bran- chial pouch anomaly and case description. Ann Otol Rhinol Laryngol. 2015;124:947-52.

2. Ahn D, SohnF J, Kim H, Yeo C. Clinical and microbiological differences between pyriform sinus fistulae in pediatric and non pediatric patients. Auris Nasus Larynx. 2015;42:34-8.

3. Nicoucar K, Giger R, Pope H, Jaecklinc T, Dulguerov P. Management of congenital fourth branchial arch anomalies: A review and analysis of published cases. J Pediatr Surg. 2009;44:1432-9.
4. Goff C, Allred C, Glade R. Current management of congenital branchial cleft cysts, sinuses and fistulae. Curr Opin Otolaryngol Head Neck Surg. 2012;20:533-9.

5. Chauhan N, Sharma Y, Bhagra T, Sud B. Branchial fistula from pyriform fossa: CT diagnosis of a case and discussion of radiological features. Clin Imaging. 2012;36:591-4.

6. Sheng Q, Lv Z, Xiao X, Zheng S, Huang Y, Huang X, et al. Diagnosis and management of pyriform sinus fistula: Experience in 48 cases. J Pediatr Surg. 2014;49:455-9.

7. Chen E, Inglis AF, Ou H, Perkins JÁ, Sie KC, Chiara J, et al. Endoscopic electrocauterization of pyriform fossa sinus tracts as definitive treatment. Int J Pediatr Otorhinolaryngol. 2009;73:1151-6. [LE

8. Lachance S, Chadha N. Systematic review of endoscopic obliteration techniques for managing congenital piriform fossa sinus tracts in children. Otolaryngol Head Neck Surg. 2016;154:241-6.

9. Givens D, Buchmann L, Park A. A novel surgical management of hypopharyngeal branchial anomalies. Int J Pediatr Otorhino- laryngol. 2015;79:579-83.

10. Arunachan P, Vaidyanathan V, Sengottan P. Open and endoscopic management of fourth branchial pouch sinus - our experience. Int Arch Otorhinolaryngol. 2015;19:309-13. 